Paul Dowling · Kevin Culligan · Kay Ohlendieck

\title{
Distal mdx muscle groups exhibiting up-regulation of utrophin and rescue of dystrophin-associated glycoproteins exemplify a protected phenotype in muscular dystrophy
}

Received: 13 March 2001 / Accepted: 19 November 2001 / Published online: 16 January 2002

(C) Springer-Verlag 2002

\begin{abstract}
Unique unaffected skeletal muscle fibres, unlike necrotic torso and limb muscles, may pave the way for a more detailed understanding of the molecular pathogenesis of inherited neuromuscular disorders and help to develop new treatment strategies for muscular dystrophies. The sparing of extraocular muscle in Duchenne muscular dystrophy is mostly attributed to the special protective properties of extremely fast-twitching smalldiameter fibres, but here we show that distal muscles also represent a particular phenotype that is more resistant to necrosis. Immunoblot analysis of membranes isolated from the well established dystrophic animal model mdx shows that, in contrast to dystrophic limb muscles, the toe musculature exhibits an up-regulation of the autosomal dystrophin homologue utrophin and a concomitant rescue of dystrophin-associated glycoproteins. Thus distal $\mathrm{mdx}$ muscle groups provide a cellular system that naturally avoids myofibre degeneration which might be useful in the search for naturally occurring compensatory mechanisms in inherited skeletal muscle diseases.
\end{abstract}

\section{Introduction}

Duchenne muscular dystrophy (DMD) represents the most common inherited neuromuscular disorder and eventually affects almost all major muscle groups. Early muscle weakness involves more proximal than distal fibres and initially alters mostly the muscle of the lower extremities and torso. At an intermediate stage, when ambulation is lost, DMD patients can still conduct limited muscle movements with their forearms and hand muscles. Although end-stage DMD also severely weakens hand muscles, a graded dystrophic response of individual

P. Dowling $\cdot$ K. Culligan $\cdot$ K. Ohlendieck $(\varangle)$

Department of Pharmacology,

Conway Institute of Biomolecular and Biomedical Research,

University College Dublin, Belfield, Dublin 4, Ireland

e-mail: kay.ohlendieck@ucd.ie

Tel.: +353-1-7161503, Fax: +353-1-2692749 groups of skeletal muscle fibres clearly exists during the natural time course of this genetic disorder (Engel et al. 1994). In contrast to a wealth of information published on the sparing of fast-twitching and small-diameter extraocular DMD muscle fibres (Andrade et al. 2000), the adaptive processes involved in the prolonged protection of more distal muscle cells from $\mathrm{Ca}^{2+}$-induced myonecrosis are not well understood.

In order to address this question, we performed a comparative immunoblot analysis of leg and toe muscle specimens from the naturally occurring dystrophic $\mathrm{mdx}$ mouse, an established model system of DMD pathology (Allamand and Campbell 2000). The suitability of mdx muscle fibres for research purposes is attested by its wide application in testing novel therapeutic strategies to ameliorate the symptoms of muscular dystrophy (Badalamente and Stracher 2000; Wehling et al. 2001). In analogy to DMD patients, the mdx skeletal muscle fibres are missing the Dp427 isoform of a protein named dystrophin due to primary genetic abnormality (Sicinski et al. 1989). The absence of this membrane cytoskeletal component causes the loss of several sarcolemmal glycoproteins, which is postulated to interrupt the proper linkage between the extracellular matrix and the membrane cytoskeleton, thereby rendering muscle cells more susceptible to necrosis (Ohlendieck 1996). Dystrophin-associated glycoproteins are represented by various isoforms of peripheral proteins termed dystroglycans, sarcoglycans, syntrophins, dystrobrevins and sarcospan (Culligan et al. 1998).

Limb muscle fibres from mdx mice exhibit segmental necrosis, are more susceptible to stretch-induced injury and osmotic shock, and show $\mathrm{Ca}^{2+}$-induced net degradation of muscle proteins (Allamand and Campbell 2000). In both mdx and DMD leg muscle specimens, it has been demonstrated that deficiency in Dp427 does not trigger a general degradation of surface glycoproteins, but specifically causes a reduction in dystrophin-associated glycoproteins (Ohlendieck 1996). Therefore this study measured the relative expression levels of dystroglycans and sarcoglycans in the microsomal membrane fraction, 
which gives a good indication of the dystrophic status of a particular group of skeletal muscle fibres.

\section{Materials and methods}

Dystrophin-deficient mdx mice, which are missing the Dp427 dystrophin isoform due to a point mutation in exon 23 (Sicinski et al. 1989), were obtained from Jackson Laboratory (Bar Harbor, Me.). In order to compare the relative expression levels of members of the dystrophin-glycoprotein complex in mdx leg and toe muscles by immunoblotting, established protocols for the isolation of microsomal membranes were employed (Ohlendieck et al. 1991). Since toe muscle fibres represent only a minute amount of tissue, four preparations of skeletal muscles pooled from ten animals each time were used in our analysis. Gel electrophoretic separation, transfer of proteins to nitrocellulose sheets, incubation with primary and secondary antibodies, as well as enhanced chemiluminescence, were carried out by previously optimised methods (Culligan et al. 2001). Antibodies were obtained from Novocastra Laboratories, Newcastle upon Tyne, UK (mAb NCL-43 to $\beta$-dystroglycan and mAb DYS-2 to the carboxy-terminus of Dp427), Upstate Biotechnology, Lake Placid, N.Y. (mAb VIA4 $4_{1}$ to $\alpha$-dystroglycan) and Affinity Bioreagents, Golden, Colo. (mAb 20A to the $\alpha_{2}$-dihydropyridine receptor). A polyclonal antibody to $\alpha$-sarcoglycan was characterised as previously described (Culligan et al. 2001) and a polyclonal antibody directed against the carboxy-terminal domain of full-length utrophin was a generous gift from S. Winder (University of Glasgow). Densitometric scanning of immuno-decorated blots was performed on a Molecular Dynamics 300 S computing densitometer (Sunnyvale, Calif.) with ImageQuant V3.0 software. Peroxidase-conjugated secondary antibodies were purchased from Boehringer Mannheim (Lewis, East Sussex), Immobilon-P nitrocellulose was from Millipore Corporation (Bedford, Mass.), and all other chemicals were of analytical grade and purchased from Sigma Chemical (Poole, Dorset). Tissue preparation, cryo-sectioning, histochemical staining and microscopy were performed as previously described in detail by Ohlendieck and Campbell (1991).

\section{Results}

A well established feature of deficiency in the sub-sarcolemmal component dystrophin is a drastic reduction in numerous muscle surface glycoproteins. However, as illustrated in Fig. 1, our comparative immunoblot analysis demonstrates that this secondary effect on the relative expression of dystrophin-associated glycoproteins does not occur in mdx toe muscle as compared with leg muscle. Although both tissues are lacking the dystrophin isoform Dp427 (Fig. 1a), the expression levels of $\alpha$-sarcoglycan (Fig. 1e) and $\alpha / \beta$-dystroglycan (Fig. 1f, g) are relatively comparable to normal muscle fibre. Importantly, the autosomal dystrophin-homologue named utrophin is up-regulated in mdx toe muscles (Fig. 1b). Laminin expression was not found to be markedly changed in the various muscle specimens studied (Fig. 1d). For control purposes, the relative expression levels of the $\alpha_{2}$-subunit of the dihydropyridine receptor are shown (Fig. 1c). Similar amounts of this highly glycosylated marker protein of the muscle cell periphery were found in microsomes isolated from normal, mdx leg and mdx toe muscle homogenates. Thus the observed differences in the relative density of dystrophin-associated glycoproteins be-

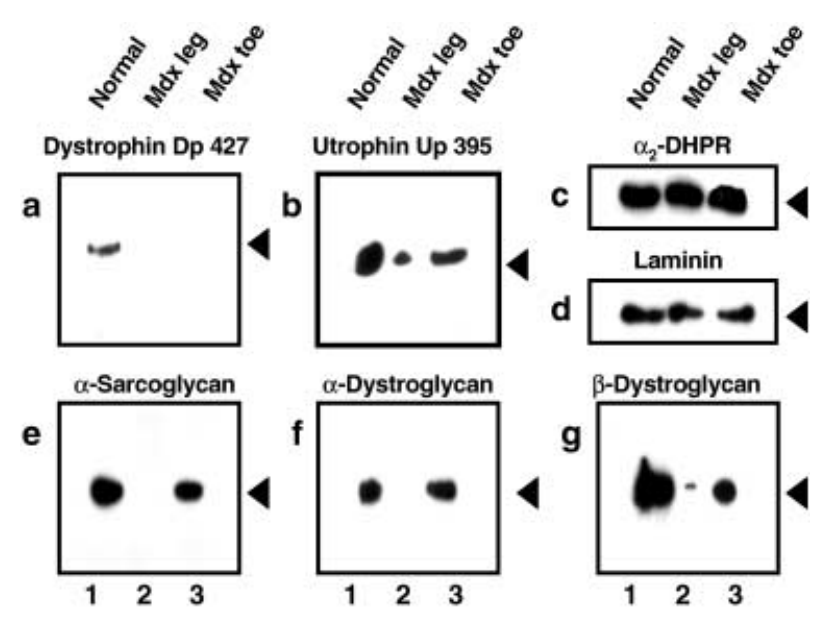

Fig. 1a-g Up-regulation of utrophin and rescue of dystrophinassociated glycoproteins in dystrophin-deficient $\mathrm{mdx}$ toe muscle, as demonstrated by comparative immunoblot analysis of microsomes isolated from normal mouse skeletal muscle (lane 1), $\mathrm{mdx}$ leg muscle (lane 2), and mdx toe muscle (lane 3). Shown are identical nitrocellulose blots labelled with antibodies to the Dp427 isoform of dystrophin (a), the Up395 isoform of utrophin (b), the $\alpha_{2}$-subunit of the dihydropyridine receptor $\left(\alpha_{2}\right.$-DHPR) $(\mathbf{c})$, laminin $(\mathbf{d})$, $\alpha$-sarcoglycan (e), $\alpha$-dystroglycan (f), and $\beta$-dystroglycan (g). In panels (a) to (g), the expression of $\alpha_{2}$-DHPR was used for internal standardisation of the immunoblotting procedure. Immuno-decorated protein bands are marked by arrow heads
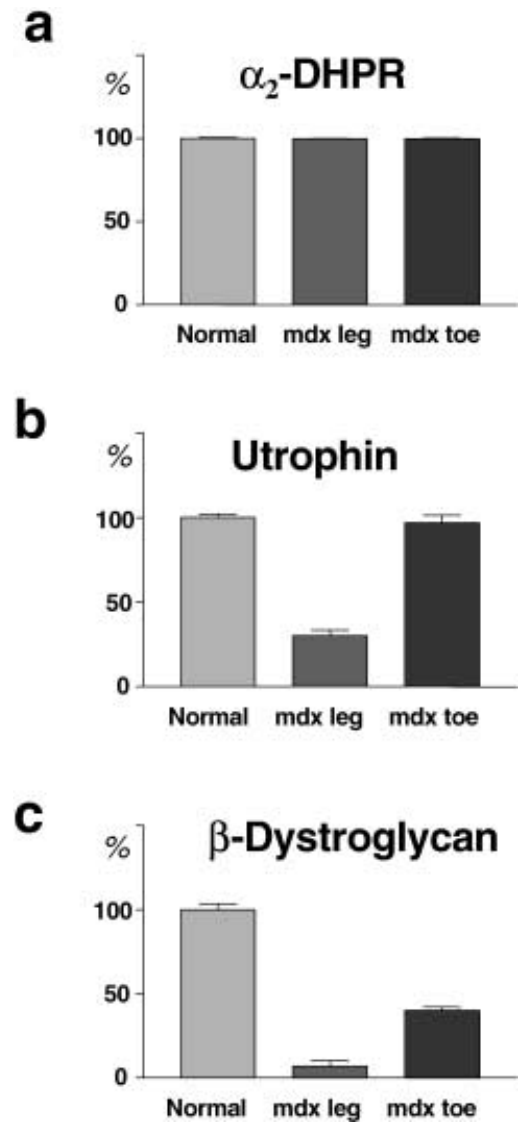

Fig. 2a-c Relative expression of utrophin, dystroglycan and the dihydropyridine receptor in normal, mdx leg and mdx toe muscle fibres. Shown is the densitometric analysis of the immuno-decoration of the $\alpha_{2}$-subunit of the dihydropyridine receptor $\left(\alpha_{2}\right.$-DHPR) (a), the Up395 isoform of utrophin (b) and $\beta$-dystroglycan (c) $(n=4)$ 

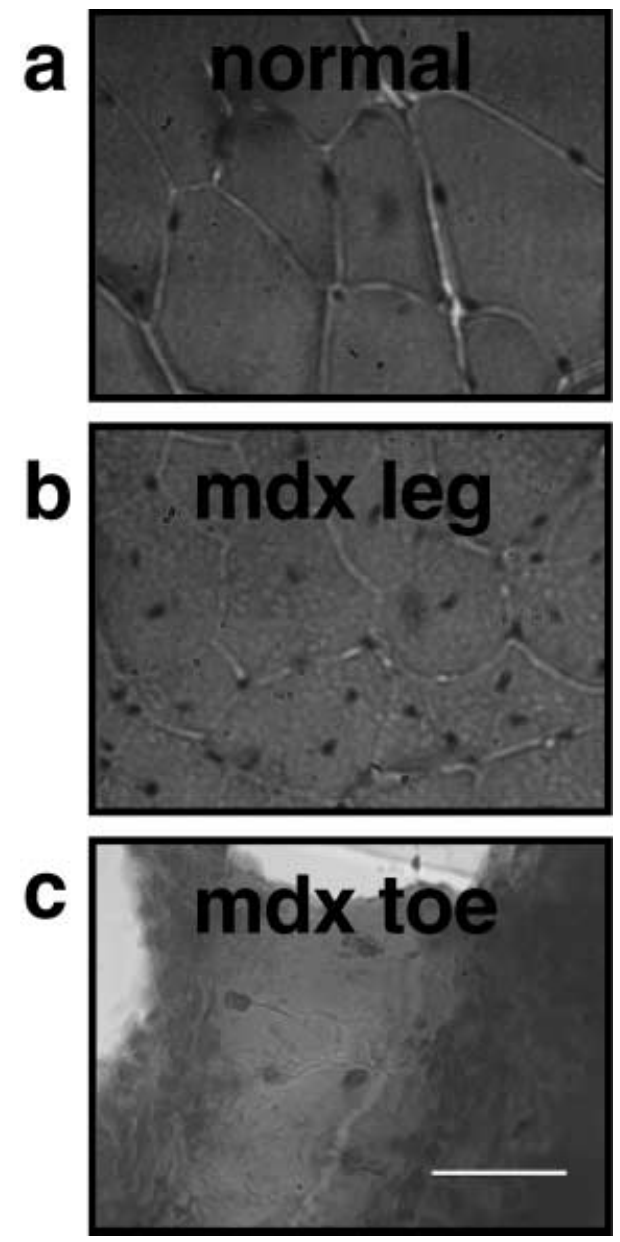

Fig. 3 Histochemical analysis of normal (a), mdx leg (b) and mdx toe (c) muscle fibres. Shown are $10 \mu \mathrm{m}$-thick transverse cryosections labelled with haematoxylin and eosin. While normal control muscle fibres (a) and mdx toe cells (c) exhibit almost exclusively peripheral nuclei, mdx leg muscle fibres show many central nuclei (b). Counting of histochemically stained nuclei in 30 fibres revealed approximately $10 \%$ peripheral nuclei in mdx leg muscle versus approximately $95 \%$ peripheral nuclei in $\mathrm{mdx}$ toe cells. Thus, mdx toe muscle, although lacking the dystrophin isoform Dp427, does not appear to show the same degree of degeneration as seen in mdx leg muscle fibres. Bar equals $20 \mu \mathrm{m}$

tween mdx leg and toe muscle groups is a reliable measurement and is not a result of the subcellular fractionation methodology. As shown in Fig. 2, the densitometric analysis of the immuno-decoration of utrophin and $\beta$-dystroglycan shows the up-regulation of utrophin and partial rescue of dystroglycan expression. The immunodecoration intensity of the dihydropyridine receptor appears to be unchanged between normal, mdx leg and mdx toe microsomes (Fig. 2a). This demonstrates that the different expression levels of utrophin and dystrophin-associated glycoproteins in the proximal versus distal limb muscles of the mdx mouse model are not an artefact of the membrane isolation procedure, differential proteolysis, protein solubilisation, electrophoretic separation and/or the immunoblotting technique.

To investigate whether the above described change in the expression levels of utrophin and dystrophin-associ- ated glycoproteins influence the cytopathological status of mdx toe muscle fibres, a comparative histochemical analysis was performed. Haematoxylin and eosin staining clearly revealed the difference between peripheral muscle fibre nuclei in normal tissue (Fig. 3a) versus central muscle fibre nuclei in the mdx leg (Fig. 3b). The observation that $\mathrm{mdx}$ toe fibres show mostly peripheral nuclei (Fig. 3c) is in agreement with the notion that the change in the relative density of dystrophin-associated glycoproteins might rescue the dystrophic phenotype in distal muscle groups. This strongly indicates that these muscle cells, although lacking the dystrophin isoform Dp427, do not exhibit the same degree of degeneration seen in mdx leg muscle fibres.

\section{Discussion}

In comparison with the established sparing of extraocular muscles, we can show here that a critical factor in the prevention of necrosis in distal muscle fibres appears to be the rescue of dystrophin-associated glycoproteins by a compensatory over-expression of utrophin. In normal skeletal muscle fibres, utrophin is highly enriched at the neuromuscular junction and co-localises with the nicotinic acetylcholine receptor complex (Ohlendieck et al. 1991). However, we were able to show previously that this protein is localised extra-junctionally in mdx fibres (Matsumura et al. 1992), so utrophin might anchor dystrophin-associated glycoproteins in dystrophin-deficient cells. This in turn might reinstate sarcolemmal integrity by at least partially re-linking the extracellular matrix to the actin membrane cystoskeleton and thereby preventing $\mathrm{Ca}^{2+}$-induced protein degradation. That the endogenous up-regulation of utrophin protects the sarcolemmal integrity has been demonstrated by severe dystrophic changes in extraocular mdx-utrn ${ }^{-/}$mouse muscle, which lacks both dystrophin and utrophin (Porter et al. 1998).

The finding that distal mdx muscle groups, although deficient in dystrophin, exemplify a protected phenotype, might be of potential significance for both diagnostic and therapeutic purposes. Due to the complexity of the clinical manifestation of inherited muscle diseases, current molecular DMD diagnostics still give occasionally ambiguous results (Miller and Hoffman 1994). Novel strategies for improving the diagnosis of DMD include systematic newborn creatine kinase screening, extended genetic testing covering gene duplications, missense mutations and stop-codon mutations, besides the more common deletions of the DMD gene, as well as carrier testing. Comparing test results for creatine kinase activity between normal muscle, dystrophic mdx leg fibres and protected mdx specimens from the extraocular or toe region might be helpful in refining this diagnostic enzyme procedure. Current therapeutic approaches for DMD include gene therapy (Hartigan-O'Connor and Chamberlain 2000), cell therapy (Partridge and Davies 1995) and pharmacological intervention (Badalamente and Stracher 2000). Important issues for the clinical application of gene therapy are the selection of the opti- 
mum delivery system, controlling expression levels without causing immunological and/or cytotoxic side effects and how genes can be sufficiently delivered to all affected cell types (Hartigan-O'Connor and Chamberlain 2000). Potential pharmacological strategies include the drug-induced up-regulation of compensatory molecules such as utrophin, the targeted introduction of protease inhibitors such as carnitine-linked leupeptin in order to inactivate $\mathrm{Ca}^{2+}$-dependent calpain activity, and immunosuppressive therapy. Especially with respect to the idea that up-regulation of utrophin prevents dystrophic changes (Rafael et al. 1998), further investigations into the naturally protected distal mdx muscle groups could help in designing the most favourable gene to be delivered into DMD muscle, whether that be by adenoviral vectors, intravascular delivery of naked DNA or stem cell transfer therapy.

In conclusion, this report demonstrates that distal $\mathrm{mdx}$ muscle groups represent unique relatively unaffected skeletal muscle fibres. In contrasting the molecular pathogenesis of necrotic mdx limb muscle with the apparently protected toe fibres, further more refined investigations into these phenotypic differences may pave the way for a more detailed understanding of DMD. Once compensatory cellular mechanisms are better understood, such pathobiochemical knowledge might help in developing new treatment strategies for muscular dystrophies. It remains to be determined whether other cellular factors, besides utrophin up-regulation, such as a lower mechanical stress per unit surface membrane area and/or metabolic differences, are also involved in the sparing of distal mdx muscle fibres.

Acknowledgements Research was supported by project grants from the Irish Health Research Board (HRB-RP-01/2001) and Enterprise Ireland, Dublin (SC/2000/386) and a European travel grant from the Royal Society, London and the Royal Irish Academy, Dublin. The authors would like to thank S.J. Winder (University of Glasgow) for providing our laboratory with antibodies.

\section{References}

Allamand V, Campbell KP (2000) Animal models for muscular dystrophy: valuable tools for the development of therapies. Hum Mol Genet 9:2459-2467
Andrade FH, Porter JD, Kaminski HJ (2000) Eye muscle sparing by the muscular dystrophies: lessons to be learned? Microsc Res Tech 48:192-203

Badalamente WA, Stracher A (2000) Delay of muscle degeneration and necrosis in mdx mice by calpain inhibition. Muscle Nerve 23:106-111

Culligan K, Mackey A, Finn D, Maguire PB, Ohlendieck K (1998) Role of dystrophin isoforms and associated glycoproteins in muscular dystrophy (review). Int J Mol Med 2:639-648

Culligan K, Glover L, Dowling P, Ohlendieck K (2001) Brain dystrophin-glycoprotein complex: persistent expression of betadystroglycan, impaired oligomerization of Dp71 and up-regulation of utrophins in animal models of muscular dystrophy. BMC Cell Biol 2:2 (http://www.biomedcentral.com/14712121/2/2)

Engel AG, Yamamoto M, Fischbeck KH (1994) Dystrophinopathies. In: Engel AG, Franzini-Armstrong C (eds) Myology: basic and clinical. McGraw-Hill, New York, pp 1133-1187

Hartigan-O'Connor D, Chamberlain JS (2000) Developments in gene therapy for muscular dystrophy. Microsc Res Tech 48:223-238

Matsumura K, Ervasti JM, Ohlendieck K, Kahl SD, Campbell KP (1992) Association of dystrophin-related protein with dystrophin-associated proteins in $\mathrm{mdx}$ mouse muscle. Nature 360: $588-591$

Miller RG, Hoffman EP (1994) Molecular diagnosis and modern management of Duchenne muscular dystrophy. Neurol Clin 12:699-725

Ohlendieck K (1996) Towards an understanding of the dystrophin-glycoprotein complex: linkage between the extracellular matrix and the subsarcolemmal membrane cytoskeleton. Eur J Cell Biol 69:1-10

Ohlendieck K, Campbell KP (1991) Dystrophin-associated proteins are greatly reduced in skeletal muscle from $\mathrm{mdx}$ mice. $\mathrm{J}$ Cell Biol 115:1685-1694

Ohlendieck K, Ervasti JM, Matsumura K, Kahl SD, Leveille CJ, Campbell KP (1991) Dystrophin-related protein is localized to neuromuscular junctions of adult skeletal muscle. Neuron 7:499-508

Partridge TA, Davies KE (1995) Myobast-based gene therapy. Br Med Bull 51:123-137

Porter JD, Rafael JA, Ragusa RJ, Brueckner JK, Trickett JI, Davies KE (1998) The sparing of extraocular muscle in dystrophinopathies is lost in mice lacking utrophin and dystrophin. J Cell Sci 111:1801-1811

Rafael JA, Tinsley JM, Potter AC, Deconinck AE, Davies KE (1998) Skeletal muscle-specific expression of utrophin transgene rescues utrophin-dystrophin deficient mice. Nat Genet 19:79-82

Sicinski P, Geng Y, Ryder-Cook AS, Barnard EA, Darlison MG, Barnard PJ (1989) The molecular basis of muscular dystrophy in the mdx mouse: a point mutation. Science 244:1578-1580

Wehling M, Spencer MJ, Tidball JG (2001) A nitric oxide synthase transgene ameliorates muscular dystrophy in $\mathrm{mdx}$ mice. J Cell Biol 155:123-31 